\title{
Coherent Transport through Systems of Coupled Quantum Dots
}

\author{
P. TROCHA ${ }^{a}$ AND J. BARNAŚ ${ }^{a, b}$ \\ ${ }^{a}$ Department of Physics, Adam Mickiewicz University \\ Umultowska 85, 61-614 Poznań, Poland \\ ${ }^{b}$ Institute of Molecular Physics, Polish Academy of Sciences \\ Smoluchowskiego 17, 60-179 Poznań, Poland
}

\begin{abstract}
Spin-dependent electronic transport through two coupled single-level quantum dots attached to ferromagnetic leads with parallel and antiparallel magnetizations is analyzed theoretically. The intra-dot Coulomb correlation is taken into account, while the inter-dot Coulomb repulsion is omitted. Conductance and tunnel magnetoresistance associated with magnetization rotation are calculated by the nonequilibrium Green function technique. The relevant Green functions are derived by the equation of motion method in the Hartree-Fock approximation. The dot occupation numbers and the Green functions are calculated self-consistently. The interference effects in electronic transport through quantum dots are analyzed in two different configurations. It is shown that the Fano resonance in conductance can be observed even for vanishing inter-dot hopping parameter $t$. The interplay of the interference effects and the Coulomb interactions in quantum dots is also analyzed.
\end{abstract}

PACS numbers: 73.23.-b, 73.21.La, 73.23.Hk

\section{Introduction}

Electronic transport through two coupled quantum dots (QDs) connected to two nonmagnetic electrodes was extensively studied in the past few years. As concerns charge transport through two quantum dots attached to magnetic leads, only a couple of papers have addressed this problem so far. Significant efforts, both theoretical [1-10] and experimental [11-14], have been undertaken recently to understand the interference effects in transport through various QD systems. These effects appear in conductance as the Fano antiresonance lines [15]. Only a few theoretical works have addressed so far the role of the signs of dot-lead non-diagonal matrix elements [16-18]. As far as we know, this issue has been 
considered only in the case of nonmagnetic leads, while no study has been carried out for quantum dots attached to magnetic leads.

Transport characteristics of the system are calculated using the Green function formalism. Since the systems with Coulomb interaction cannot be treated exactly, we applied the Hartree-Fock decoupling scheme to calculate the higher order Green functions from the relevant equations of motion. The average values of the occupation numbers (which enter expressions for the Green functions) have been calculated self-consistently.

The paper is organized as follows. In Sect. 2 we present the model and briefly describe the theoretical method used to calculate the transport characteristics. Numerical results on the conductance and associated tunnel magnetoresistance (TMR) are presented and discussed in Sect. 3.

\section{Model and analytical solution}

In this paper we consider two coupled single-level quantum dots. The dots are connected to ferromagnetic leads as shown schematically in Fig. 1. For simplicity, magnetic moments of the leads are assumed to be either parallel or antiparallel.
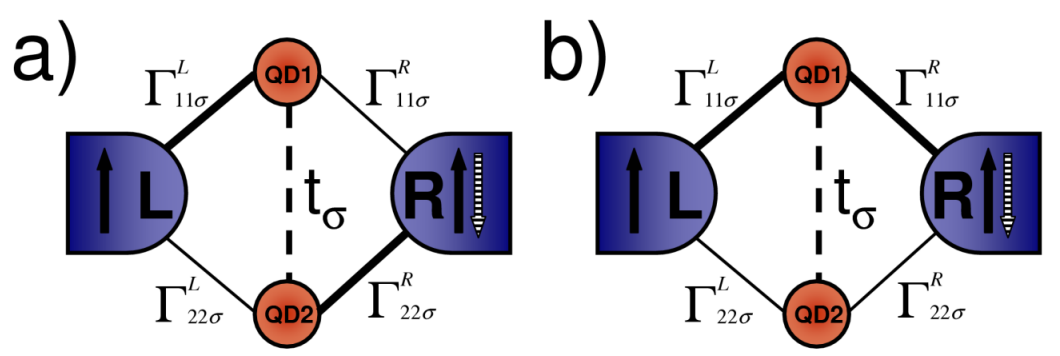

Fig. 1. Schematic picture of the two configurations of a DQD system coupled to ferromagnetic leads studied in this paper. The parameter $\Gamma_{i i \sigma}^{\alpha}(\alpha=\mathrm{L}, \mathrm{R}, i=1,2)$ describes here a contribution to the spin dependent level width due to coupling of the $i$-th dot to the $\alpha$-th lead. Thickness of the solid lines symbolically reflects strength of the corresponding coupling.

We assume that the inter-dot Coulomb interaction is negligible in comparison with the intra-dot one, and therefore has been omitted. The system can be then described by Hamiltonian of the general form

$$
\hat{H}=\hat{H}_{\text {leads }}+\hat{H}_{\mathrm{DQD}}+\hat{H}_{\text {tunnel }},
$$

where the first term, $\hat{H}_{\text {leads }}$, describes the left $(\mathrm{L})$ and right $(\mathrm{R})$ electrodes in the non-interacting quasi-particle approximation, $\hat{H}_{\text {leads }}=\hat{H}_{\mathrm{L}}+\hat{H}_{\mathrm{R}}$, with $\hat{H}_{\alpha}=\sum_{\boldsymbol{k} \sigma} \epsilon_{\boldsymbol{k} \alpha \sigma} c_{\boldsymbol{k} \alpha \sigma}^{\dagger} c_{\boldsymbol{k} \alpha \sigma}$ (for $\left.\alpha=\mathrm{L}, \mathrm{R}\right)$. Here, $c_{\boldsymbol{k} \alpha \sigma}^{\dagger}\left(c_{\boldsymbol{k} \alpha \sigma}\right)$ is the creation (annihilation) operator of an electron with the wave vector $\boldsymbol{k}$ and spin $\sigma$ in the lead $\alpha$, whereas $\epsilon_{\boldsymbol{k} \alpha \sigma}$ denotes the corresponding single-particle energy. 
The second term of the Hamiltonian (1) describes the two coupled quantum dots,

$$
\hat{H}_{\mathrm{DQD}}=\sum_{i \sigma} \epsilon_{i \sigma} d_{i \sigma}^{\dagger} d_{i \sigma}-t \sum_{\sigma}\left(d_{1 \sigma}^{\dagger} d_{2 \sigma}+\text { h.c. }\right)+\sum_{i} U_{i} n_{i \sigma} n_{i \bar{\sigma}},
$$

where $\bar{\sigma} \equiv-\sigma, n_{i \sigma}=d_{i \sigma}^{\dagger} d_{i \sigma}$ is the particle number operator, $\epsilon_{i \sigma}$ is the discrete energy level of the $i$-th dot, $t$ is the inter-dot hopping parameter (assumed real and independent of the electron spin orientation), whereas $U_{i}(i=1,2)$ is the Coulomb integral for two electrons residing on the $i$-th dot. In a general case, the dot levels can be spin dependent.

The last term, $H_{\text {tunnel }}$, of the Hamiltonian (1) describes electron tunneling between the leads and dots, and takes the form

$$
\hat{H}_{\text {tunnel }}=\sum_{\boldsymbol{k} \alpha} \sum_{i \sigma}\left(V_{i \boldsymbol{k} \sigma}^{\alpha} c_{\boldsymbol{k} \alpha \sigma}^{\dagger} d_{i \sigma}+\text { h.c. }\right) \text {, }
$$

where $V_{i \boldsymbol{k} \sigma}^{\alpha}$ are the relevant matrix elements. Coupling of the dots to external leads can be parameterized in terms of $\Gamma_{i j \sigma}^{\alpha}(\epsilon)=2 \pi \sum_{\boldsymbol{k}} V_{i \boldsymbol{k} \sigma}^{\alpha} V_{j \boldsymbol{k} \sigma}^{\alpha *} \delta\left(\epsilon-\epsilon_{\boldsymbol{k} \alpha \sigma}\right) \approx$ $2 \pi V_{i \sigma}^{\alpha} V_{j \sigma}^{\alpha *} \rho_{\alpha}$, with $\rho_{\alpha}$ being the density of states in the $\alpha$-th lead. We assume that $\Gamma_{i j \sigma}^{\alpha}$ is constant within the energy band, $\Gamma_{i j \sigma}^{\alpha}(\epsilon)=\Gamma_{i j \sigma}^{\alpha}=$ const for $\epsilon \in\langle-D, D\rangle$, and $\Gamma_{i j \sigma}^{\alpha}(\epsilon)=0$ otherwise. Here, $2 D$ denotes the electron band width.

In this paper we consider two arrangements of the couplings between the dots and leads in the parallel configuration: (i) $\Gamma_{11 \sigma}^{\mathrm{L}}=\Gamma_{0}(1 \pm p), \Gamma_{12 \sigma}^{\mathrm{L}}=\Gamma_{21 \sigma}^{\mathrm{L}}=$ $\Gamma_{0} \sqrt{\beta}(1 \pm p), \Gamma_{22 \sigma}^{\mathrm{L}}=\beta \Gamma_{0}(1 \pm p), \Gamma_{11 \sigma}^{\mathrm{R}}=\beta \Gamma_{0}(1 \pm p), \Gamma_{12 \sigma}^{\mathrm{R}}=\Gamma_{21 \sigma}^{\mathrm{R}}=\Gamma_{0} \sqrt{\beta}(1 \pm p)$, and $\Gamma_{22 \sigma}^{\mathrm{R}}=\Gamma_{0}(1 \pm p)$ for $\sigma=\uparrow$ (upper sign) and $\sigma=\downarrow$ (lower sign), where $p$ is the polarization factor of the leads, $\Gamma_{0}$ is a constant, and $\beta$ takes into account difference in the coupling of a given electrode to the two dots; (ii) $\Gamma_{11 \sigma}^{\mathrm{L}}=\Gamma_{0}(1 \pm p)$, $\Gamma_{12 \sigma}^{\mathrm{L}}=\Gamma_{21 \sigma}^{\mathrm{L}}=q_{\mathrm{L}} \Gamma_{0} \sqrt{\beta}(1 \pm p), \Gamma_{22 \sigma}^{\mathrm{L}}=\beta \Gamma_{0}(1 \pm p), \Gamma_{11 \sigma}^{\mathrm{R}}=\Gamma_{0}(1 \pm p), \Gamma_{12 \sigma}^{\mathrm{R}}=\Gamma_{21 \sigma}^{\mathrm{R}}=$ $q_{\mathrm{R}} \Gamma_{0} \sqrt{\beta}(1 \pm p)$, and $\Gamma_{22 \sigma}^{\mathrm{R}}=\beta \Gamma_{0}(1 \pm p)$. In the case (ii) the factors $q_{\mathrm{L}}$ and $q_{\mathrm{R}}$ take into account signs of $\Gamma_{12 \sigma}^{\mathrm{L}}$ and $\Gamma_{12 \sigma}^{\mathrm{R}}$, so that $q_{\mathrm{L}} / q_{\mathrm{R}}= \pm 1$. Generally, one is free to choose the signs of the off-diagonal elements of both $\Gamma_{\sigma}^{\mathrm{L}}$ and $\boldsymbol{\Gamma}_{\sigma}^{\mathrm{R}}$, because $\left(\Gamma_{i j \sigma}^{\alpha}\right)^{2}=\Gamma_{i i \sigma}^{\alpha} \Gamma_{j j \sigma}^{\alpha}$.

Assuming $\Gamma_{12 \sigma}^{\mathrm{L}} / \Gamma_{12 \sigma}^{\mathrm{R}}=1$ or $\Gamma_{12 \sigma}^{\mathrm{L}} / \Gamma_{12 \sigma}^{\mathrm{R}}=-1$ we deal with two distinct models. In the following we will refer to the first model as the plus model, for which $q_{\mathrm{L}} / q_{\mathrm{R}}=1$. On the other hand, the model with $q_{\mathrm{L}} / q_{\mathrm{R}}=-1$ will be referred to as the minus model. One can show that both dot states in the plus model are coupled to the same effective channel, i.e. to the states $c_{\boldsymbol{k} \sigma+}=$ $\left(c_{k \mathrm{~L} \sigma}+c_{\boldsymbol{k} \mathrm{R} \sigma}\right) / \sqrt{2}$, but they are completely decoupled from the antisymmetric combination $c_{\boldsymbol{k} \sigma-}=\left(c_{\boldsymbol{k} L \sigma}-c_{\boldsymbol{k} \mathrm{R} \sigma}\right) / \sqrt{2}$. In turn, in the minus model one dot is connected to the symmetric combination $\left(c_{\boldsymbol{k} \sigma+}\right.$ states) but the other is connected to the antisymmetric combination ( $c_{\boldsymbol{k} \sigma-}$ states). Thus, in the minus case the dots are connected to different effective channels [16].

Electric current $J$ flowing through the system is determined by the retarded, advanced, and lesser Green functions of the dots according to the following general formula $[19,20]$ : 


$$
\begin{aligned}
J= & \frac{\mathrm{i} e}{2 \hbar} \sum_{\sigma} \int \frac{\mathrm{d} \epsilon}{2 \pi} \operatorname{Tr}\left\{\left[\boldsymbol{\Gamma}_{\sigma}^{\mathrm{L}}-\boldsymbol{\Gamma}_{\sigma}^{\mathrm{R}}\right] \boldsymbol{G}_{\sigma}^{<}(\epsilon)\right. \\
& \left.+\left[f_{\mathrm{L}}(\epsilon) \boldsymbol{\Gamma}_{\sigma}^{\mathrm{L}}-f_{\mathrm{R}}(\epsilon) \boldsymbol{\Gamma}_{\sigma}^{\mathrm{R}}\right]\left[\boldsymbol{G}_{\sigma}^{\mathrm{r}}(\epsilon)-\boldsymbol{G}_{\sigma}^{\mathrm{a}}(\epsilon)\right]\right\} .
\end{aligned}
$$

Here, $f_{\alpha}(\epsilon)=\left[\mathrm{e}^{\left(\epsilon-\mu_{\alpha}\right) / k_{\mathrm{B}} T}+1\right]^{-1}$ is the Fermi-Dirac distribution function for the lead $\alpha, \boldsymbol{G}_{\sigma}^{<}(\epsilon)$ and $\boldsymbol{G}_{\sigma}^{\mathrm{r}(\mathrm{a})}(\epsilon)$ are the Fourier transforms of the lesser and retarded (advanced) Green functions of the dots for spin $\sigma$. To calculate the Green functions $G_{i j \sigma}^{\mathrm{r}(\mathrm{a})}(\epsilon)$, we write the corresponding equation of motion and apply the Hartree-Fock decoupling scheme for higher order Green functions. In turn, the lesser Green function $G_{i j \sigma}^{<}(\epsilon)$ has been calculated from the corresponding equation of motion, with the higher order Green functions calculated on taking into account the Langreth theorem [20] and the Hartree-Fock decoupling scheme assumed when calculating $G_{i j \sigma}^{\mathrm{r}(\mathrm{a})}(\epsilon)$. We note that the formulae for Green functions include the expectation values $\left\langle d_{i \sigma}^{\dagger} d_{i \sigma}\right\rangle, n_{i \bar{i} \sigma},\left\langle d_{i \sigma}^{\dagger} c_{k \alpha \sigma}\right\rangle$ and $\left\langle c_{k \alpha \sigma}^{\dagger} d_{i \sigma}\right\rangle$, which can be determined taking into account the identities, $n_{i \sigma}=-\mathrm{i} \int \frac{\mathrm{d} \epsilon}{2 \pi} G_{i i \sigma}^{<}(\epsilon), n_{i \bar{i} \sigma}=-\mathrm{i} \int \frac{\mathrm{d} \epsilon}{2 \pi} G_{\bar{i} i \sigma}^{<}(\epsilon)$, $\left\langle d_{i \sigma}^{\dagger} c_{k \alpha \sigma}\right\rangle=-\mathrm{i} \int \frac{\mathrm{d} \epsilon}{2 \pi}\left\langle\left\langle c_{k \alpha \sigma} \mid d_{i \sigma}^{\dagger}\right\rangle\right\rangle^{<}$, and a similar one for $\left\langle c_{k \alpha \sigma}^{\dagger} d_{i \sigma}\right\rangle$.

\section{Numerical results}

Let us consider first a noninteracting case, $U=0$. In Fig. 2 the linear conductance is plotted as a function of the position of the average bare dot's level $E=\left(\epsilon_{1}+\epsilon_{2}\right) / 2$, where the dot levels have been assumed to be spin degenerate, $\epsilon_{i \sigma}=\epsilon_{i}$ for $\sigma=\uparrow, \downarrow$, and $\epsilon_{1}=E+\Delta / 2, \epsilon_{2}=E-\Delta / 2$, with $\Delta=\epsilon_{1}-\epsilon_{2}$. To simplify notation we also introduce the quantity $q=q_{\mathrm{L}} / q_{\mathrm{R}}$.

From the numerical analysis follows that in order to observe the Fano line in conductance for the system shown in Fig. 1a one needs a nonzero inter-dot coupling, whereas for the structure shown in Fig. 1b the Fano effect appears in both models $\left(q_{\mathrm{L}} / q_{\mathrm{R}}= \pm 1\right)$ even for vanishing inter-dot coupling parameter $(t=0)$, provided the dot levels are different, $\epsilon_{1} \neq \epsilon_{2}$. However, the Fano line shapes for the two models are different.

Let us now give some insight into the two models shown in Fig. 1b. One can prove that in the minus model, the density of states for each of the dots' levels is unaffected by the other level, because they are effectively decoupled as we already mentioned above. This, however, is not true for the plus model $(q=1)$.

Generally, the interference effects in conductance can be observed in both models, despite the fact that the dots' levels do not interfere in the case of $q=-1$. This appears because the transmission takes place between the left and right leads, and not between the symmetric and antisymmetric combinations of $c_{\boldsymbol{k} \mathrm{L} \sigma}$ and $c_{\boldsymbol{k} \mathrm{R} \sigma}$. In the plus model, when one of the dots' levels $\left(\epsilon_{1}\right.$ or $\left.\epsilon_{2}\right)$ approaches the Fermi level of the leads, one observes a maximum in the conductance. The conductance goes to zero when the chemical potential of the leads coincides with $\epsilon_{+}=\left(\Gamma_{11 \sigma} \epsilon_{2}+\Gamma_{22 \sigma} \epsilon_{1}\right) /\left(\Gamma_{11 \sigma}+\Gamma_{22 \sigma}\right)\left(\right.$ where $\Gamma_{i i \sigma}=\Gamma_{i i \sigma}^{\mathrm{L}}+\Gamma_{i i \sigma}^{\mathrm{R}}$ for $\left.i=1,2\right)$. On the other hand, for the minus model, the conductance has maxima in the vicinity of the dot's levels, and reaches zero at $\epsilon_{-}=\left(\Gamma_{11 \sigma} \epsilon_{2}-\Gamma_{22 \sigma} \epsilon_{1}\right) /\left(\Gamma_{11 \sigma}-\Gamma_{22 \sigma}\right)$. When 

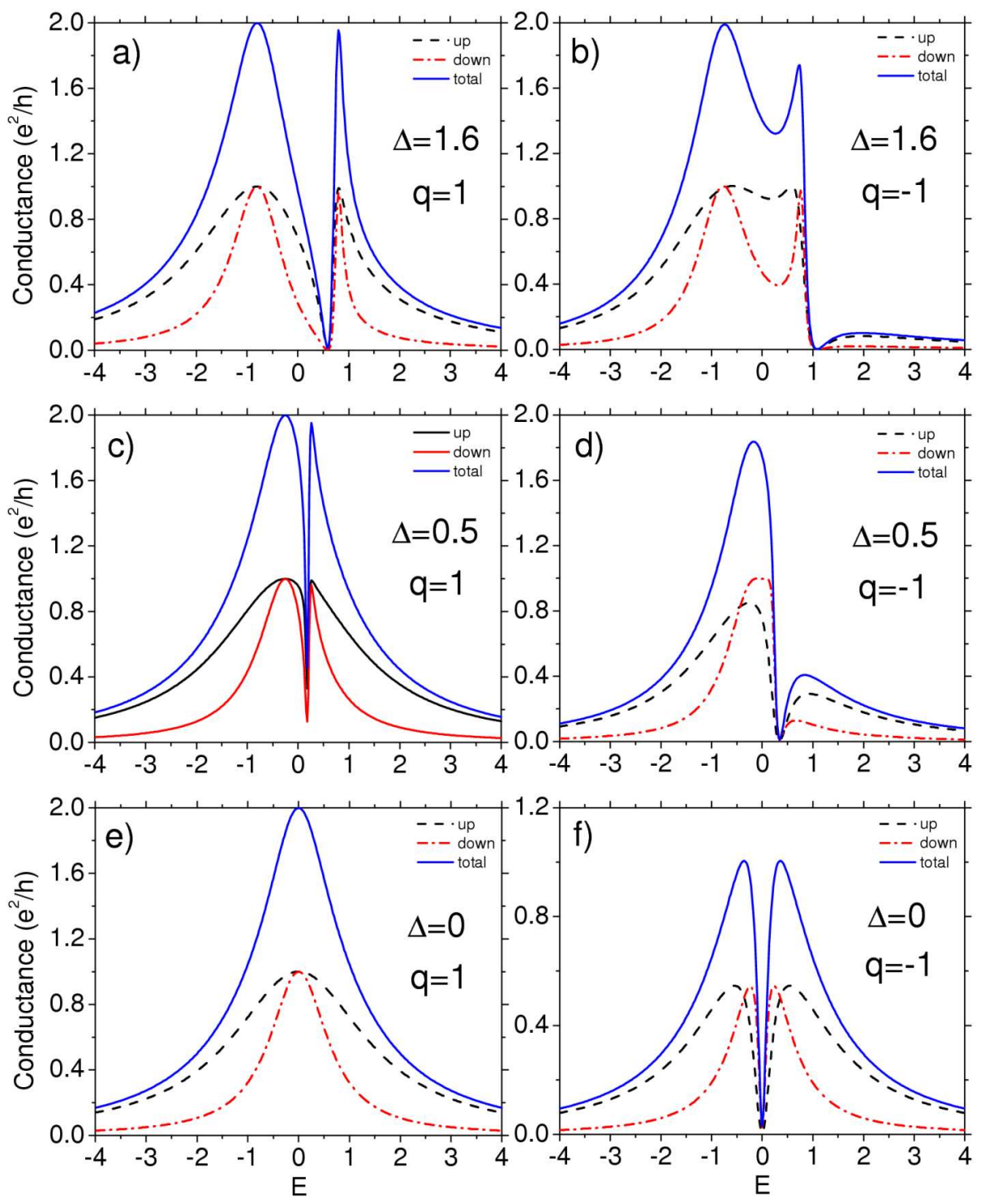

Fig. 2. Linear conductance as a function of the position of average bare dot's level for parallel magnetic configuration, and for the plus model (left part) and minus model (right part), calculated for indicated values of the splitting of the dots levels $\Delta$. The other parameters are: $p=0.4, t=0, \beta=0.15, U=0$, and $k_{\mathrm{B}} T=0.01$. The energy is measured in the units of $\Gamma$.

the dots' levels are close to each other, the two maxima merge into a single peak (see Fig. 2d). For $\epsilon_{1}=\epsilon_{2}$ one observes a dip structure, see Fig. 2f. The dip is a consequence of the destructive quantum interference of electron waves transmitted 
through the two levels of the double quantum dot (DQD) system, renormalized due to an effective interaction of the bare levels via the electrodes owing to nonzero off-diagonal elements of the coupling matrix [8]. It is worth noting that a similar situation also occurs in the model shown in Fig. 1a, when the dot levels are equal, $\epsilon_{1}=\epsilon_{2}$, inter-dot coupling parameter vanishes, $t=0$, and $\beta \rightarrow 0$. At least, for $\epsilon_{1}=\epsilon_{2}$ and $\Gamma_{11 \sigma}=\Gamma_{22 \sigma}$, a complete destructive interference takes place. Ferromagnetism of the electrodes has some influence on positions of the conductance maxima for both spin orientations, but only for the minus model. In the minus model the maxima positions (Breit-Wigner and Fano) for both spin orientations are shifted away, whereas in the plus model they are unaffected.

Figure 3 shows TMR in the linear response regime as a function of the position of average dot's level for both plus and minus models. When the dots' levels are well above or well below the Fermi level of the leads, the TMR tends to the Julliere value observed in planar magnetic tunnel junctions. One can see that in the minus model the TMR tends to Julliere's value faster than in the plus model. When, in turn, the dot levels cross the Fermi energy, the situation becomes more complex and TMR significantly depends on the sign of $q$. In the limit of $U=0$, see Fig. 3, TMR is then suppressed below the Julliere value in the minus model, whereas for the plus model this suppression occurs only in a part of this region. It is worth noting that a similar suppression of TMR was also found in single quantum dots [21]. However, the suppression of TMR in Fig. 3 is stronger than that in a single dot. Moreover, in the minus model TMR even changes sign for a certain region of the average dot's level position, while TMR in that region becomes enhanced in the plus model.

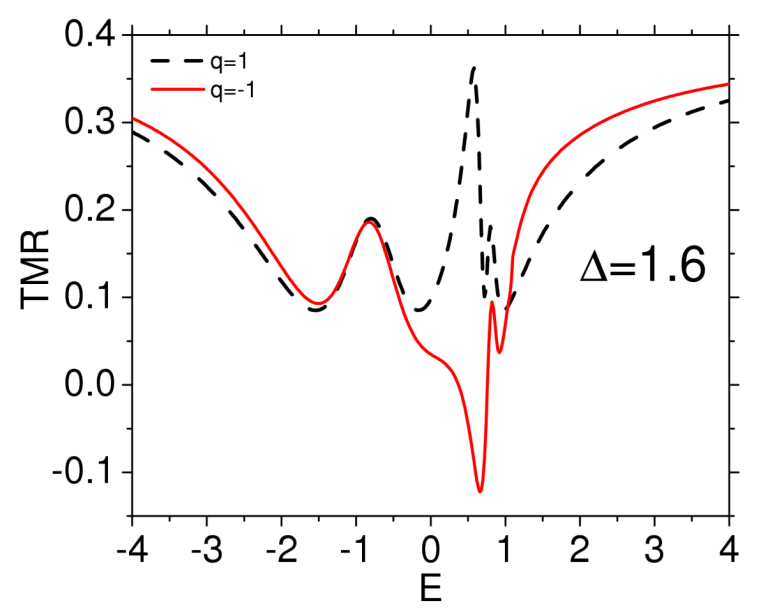

Fig. 3. TMR as a function of the position of average bare dot's level for the plus model $(q=1)$ and minus model $(q=-1)$, and for $\Delta=1.6$ and $U=0$. The other parameters are the same as in Fig. 2. 
Let us consider now the influence of the intra-dot Coulomb repulsion on the picture described above. This is shown in Fig. 3 for indicated value of the parameter $U$ and for both models. As follows from the presented numerical results, the Coulomb interaction leads to splitting of the double peak structure from Fig. 2a, b. As a consequence, the four-peak structure develops now in the conductance for finite $U$. Particularly, the four-peak structure in the total conductance (for both spin orientations) is well resolved in the plus model (Fig. 4a), whereas in the minus model one observes three peaks in the total and spin-up conductance, and four peaks in the spin-down conductance. This is because splitting of the resonances in the vicinity of $-\Delta / 2$ in the minus model is not well resolved. It is also worth noting that in the minus model, a characteristic Coulomb gap between the "original" peaks and their Coulomb counterparts appears for a sufficiently large $U$.

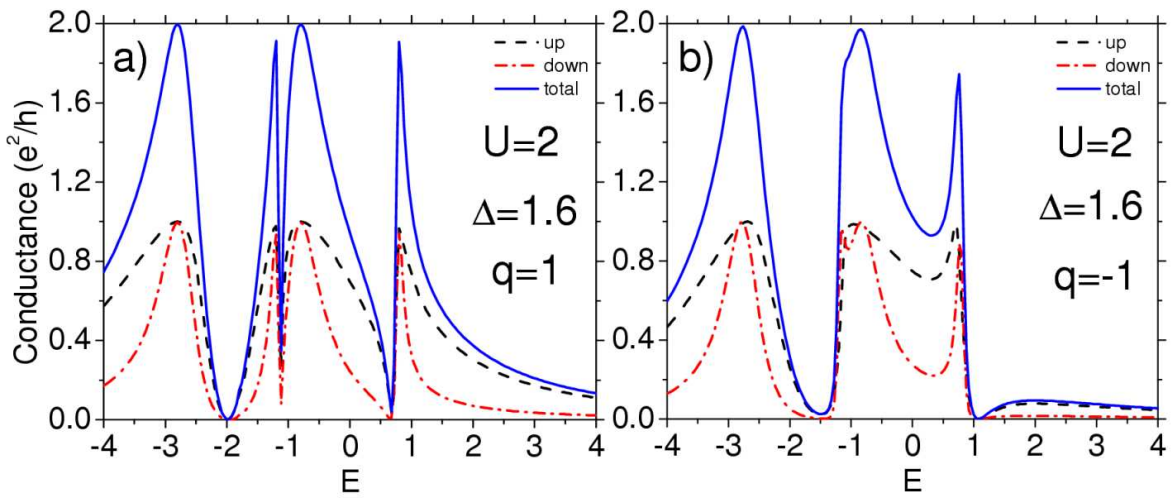

Fig. 4. Linear conductance as a function of the position of average bare dot's level for parallel magnetic configuration, and for the plus model (a) and minus model (b), calculated for $\Delta=1.6$, and $U=2$. The other parameters are: $p=0.4, t=0, \beta=0.15$, and $k_{\mathrm{B}} T=0.01$. The energy is measured in the units of $\Gamma$.

In conclusion, we have considered the interference effects in electronic transport through two quantum dots coupled to ferromagnetic leads, with two different arrangements of the couplings. We have shown that the Fano resonance may be observed even for vanishing inter-dot coupling parameter.

\section{Acknowledgments}

This work, as part of the European Science Foundation EUROCORES Programme SPINTRA, was supported by funds from the Ministry of Science and Higher Education as a research project in years 2006-2009 and the EC Sixth Framework Programme, under contract No. ERAS-CT-2003-980409. 


\section{References}

[1] M.L. Landron de Guevara, F. Claro, P.A. Orellana, Phys. Rev. B 67, 195335 (2003).

[2] H. Lu, R. Lü, B.-Fen Zhu, J. Phys., Condens. Matter 18, 8961 (2006).

[3] H. Lu, R. Lü, B.-Fen Zhu, Phys. Rev. B 71, 235320 (2005).

[4] R. Lü, Z.-R. Liu, G.-M. Zhang, cond-mat/0504288.

[5] S.Y. Cho, R.H. McKenzie, K. Kang, C.K. Kim, J. Appl. Phys., Condens. Matter 15, 1147 (2003).

[5] Guo-H. Ding, C.K. Kim, K. Nahm, Phys. Rev. B 71, 205313 (2005).

[6] P. Trocha, J. Barnaś, Phys. Status Solidi C 3, 113 (2006).

[7] P. Trocha, J. Barnaś, Phys. Status Solidi B 244, 2553 (2007).

[8] P. Trocha, J. Barnaś, to appear in Mater. Sci. (Poland).

[9] A. Wysocki, J. Barnaś, Acta Phys. Superficierum 9, 177 (2006).

[10] Y. Tanaka, N. Kawakami, Phys. Rev. B 72, 085304 (2005).

[11] J. Gores, D. Goldhaber-Gordon, S. Heemeyer, M.A. Kastner, H. Shtrikman, D. Mahalu, U. Meirav, Phys. Rev. B 62, 2188 (2000).

[12] A.A. Clerk, X. Waintal, P.W. Brouwer, Phys. Rev. Lett. 86, 4636 (2001).

[13] K. Kobayashi, H. Aikawa, S. Katsumoto, Y. Iye, Phys. Rev. Lett. 88, 256806 (2002); Phys. Rev. B 68, 235304 (2003).

[14] A.C. Johnson, C.M. Marcus, M.P. Hanson, A.C. Gossard, Phys. Rev. Lett. 93, 106803 (2004).

[15] U. Fano, Phys. Rev. 124, 1866 (1961).

[16] M. Sindel, A. Silva, Y. Oreg, J. von Delft, Phys. Rev. B 72, 125316 (2005).

[17] M. Goldstein, R. Berkovits, New J. Phys. 9, 118 (2007).

[18] A. Silva, Y. Oreg, Y. Gefen, Phys. Rev. B 66, 195316 (2002).

[19] Y. Meir, N.S. Wingreen, Phys. Rev. Lett. 68, 2512 (1992).

[20] A.P. Jauho, N.S. Wingreen, Y. Meir, Phys. Rev. B 50, 5528 (1994).

[21] I. Weymann, J. Köenig, J. Martinek, J. Barnaś, G. Schön, Phys. Rev. B 72, $115334(2005)$. 